\title{
Gestalt y Heterodoxia: un cambio proactivo aplicable en la praxis investigativa universitaria*
}

\author{
Beatriz Carolina Carvajal; Soc. PhD **
}

Recibido: 18 de enero de 2016

Evaluado: 10 de marzo de 2016

Aceptado: 1 de abril de 2016

\section{RESUMEN}

La pesquisa que da origen a este artículo está soportada en la propia experiencia en el área de sistemas humanos, específicamente en el estudio del quehacer investigativo y la gestión del conocimiento universitario latinoamericano. De esa experiencia previa, se desprende la siguiente premisa: el contexto cambiante exige a las universidades respuestas basadas en un enfoque vanguardista para comprender e interpretar los hechos sociales y naturales. El objetivo que fundamenta este reporte es proponer estrategias de acción creativas en el quehacer investigativo para el reciclaje de los métodos de investigación, y así, generar una praxis proactiva, gestáltica y heterodoxa. Los métodos aplicados fueron la investigación documental y la reflexión hermenéutica. Se tomó una muestra intencional y se realizaron entrevistas a informantes clave provenientes de diversas universidades e institutos de investigación latinoamericanos y que están afiliados a una de estas redes: 1) Red para la Difusión Científica y Humanística Heterodoxias y 2) Red de Estudios Organizacionales en América Latina, el Caribe e Iberoamérica. Como soporte teórico se reflexiona con base en los constructos de Gestalt (Perls, 1977; Moreau, 1999; Vázquez-Badin, 2010) y de Heterodoxia (Savater y Villena, 1989; Carvajal, 2010a).

El artículo de investigación constituye uno de los resultados de la investigación denominada: "Gestalt y Heterodoxia. Desde la totalidad hacia la heterodoxia en la praxis investigativa", que ha sido financiada por el Consejo de desarrollo científico, tecnológico y humanístico (CDCHT) de la Universidad Centroccidental "Lisandro Alvarado, Codificada con el número: 005-AC-2015. DOI: http://dx.doi. org/10.15332/s1794-3841.2017.0027.03

** Postdoctora egresada del Programa Multidisciplinario de Formación Continua para Doctores en Ciencias Sociales, Ciencias de la Comunicación, Humanidades y Artes, Universidad Nacional de Córdoba, Argentina, año 2008. Profesora Titular en la Universidad Centroccidental "Lisandro Alvarado". Correo electrónico: beatrizcarvaja@@ucla.edu.ve, becaro777@gmail.com. Barquisimeto, estado Lara, Venezuela. 
Se concluye que el cambio proactivo aplicable en la praxis investigativa universitaria supone la responsabilidad, el compromiso y el liderazgo del investigador universitario como agente transformacional, lo cual conlleva la revalorización de la vinculación universidad-sector socio productivo y demanda la innovación, la creatividad y la recreación permanentemente de los métodos de investigación usados para comprender e interpretar la realidad desde la heterodoxia y la totalidad.

Palabras Clave: Gestalt, heterodoxia, praxis investigativa universitaria y reflexión hermenéutica. 


\section{Gestalt and Heterodoxy: a proactive applicable change in university research praxis}

Received: January 18, 2016

Evaluated: March 10, 2016 Accepted: April 1, 2016

\section{Abstract.}

The research that gives rise to this article is supported on personal experience in the area of human systems, specifically in the study of research activities and management of the Latin American university knowledge. From that previous experience, the premise is clear: the changing context requires answers to universities based on an avant-garde approach to understanding and interpreting social and natural facts. The objective underlying this report is to propose creative strategies for action in the research work for recycling research methods, and thus generate a proactive, gestalt and heterodox practice. The methods used were documentary research and hermeneutical reflection. A purposive sample was taken and interviews were conducted with key informants from various universities and institutes of Latin American research and are affiliated with one of these networks: 1) Network for Scientific Dissemination and Humanistic heterodoxies and 2) Network of Organizational Studies in Latin America, the Caribbean and Ibero-America. As a technical support, there is a reflection based on the gestalt constructs (Perls, 1977, Moreau, 1999; Vázquez-Badin, 2010) and heterodoxy (Savater and Villena, 1989; Carvajal, 2010a). It is concluded that proactive change applicable in university research praxis assumes responsibility, commitment and leadership of the university researcher as transformational agent, which leads to the revaluation of the university-productive sector partner and demand innovation bonding, creativity and permanent recreation of the research methods used to understand and interpret reality from heterodoxy and totality.

Keywords: Gestalt, heterodoxy, university research praxis and hermeneutical reflection. 


\section{Gestalt e Heterodoxia: uma mudança proativa aplicável na práxis da pesquisa universitária}

\section{Resumo}

A pesquisa que origina este artigo está suportada na própria experiência na área de sistemas humanos, especificamente no estudo do afazer da pesquisa e a gestão do conhecimento universitário latino-americano. Desta experiência previa, se desprende a seguinte premissa: o contexto cambiante exige às universidades respostas baseadas em um enfoque vanguardista para compreender e interpretar os fatos sociais e naturais. O objetivo que fundamenta este relatório é propor estratégias de ação criativas no afazer da pesquisa para a reciclagem dos métodos de pesquisa e assim gerar uma práxis proativa, gestálica e heterodoxa.

Os métodos aplicados foram a pesquisa documental e a reflexão hermenêutica. Tomou-se uma mostra intencional e se realizaram entrevistas aos informantes chaves provenientes de diversas universidades e institutos de pesquisa latino-americanos e que estão filiados em uma destas redes: 1) Rede para a Difusão Cientifica e Humanística Heterodoxia e 2) Rede de Estudos Organizacionais na América Latina, o Caribe e Ibero-América. Como suporte teórico reflete-se baseado nos construtos de Gestalt (Perls, 1977; Moreau, 1999; Vázquez-Badin, 2010) e de Heterodoxia (Savater e Villena, 1989; Carvajal, 2010a).

Conclui-se que a mudança proativa aplicável na práxis da pesquisa universitária supõe a responsabilidade, o compromisso e a liderança do pesquisador universitário como agente transformacional, o qual implica a revalorização da vinculação universidade-setor socioprodutivo e demanda a inovação, a criatividade e a recriação permanentemente dos métodos de pesquisa usados para compreender e interpretar a realidade desde a heterodoxia e a totalidade.

Palavras-chave: Gestalt, heterodoxia, práxis da pesquisa universitária e reflexão hermenêutica.
Recebido: 18 de janeiro de 2016

Avaliado: 10 de março de 2016

Aceito: 1 de april de 2016 


\section{Presentación}

De la línea de investigación que ha marcado la praxis investigativa de quien escribe, sistemas humanos y métodos de investigación, se tomaron dos premisas básicas que constituyen los ejes de la investigación reseñada en este artículo. Estas son: a) nuestra mente observa e interpreta la realidad en movimiento fluyente, es decir, se va del todo a las partes, de la figura al fondo, en movimiento dialéctico, develando una realidad que sólo es conocida en los límites concretos de nuestra percepción (Carvajal, 2010b); y b) las universidades están llamadas a ser un espacio en el que se fomente el cambio continuo, por medio de su quehacer docente, investigativo y de extensión (Carvajal, 2001). El cómo optemos, como investigadores universitarios, atender esa exhortación no sólo marcará una diferencia en cuánto a nuestro enfoque interpretativo de una realidad, sino en cuánto al qué y para qué explicar y comprender esa realidad que solo se manifiesta en limites concretos a nuestra particular observación.

Por consiguiente, ese interés concreto en seguir indagando acerca de la praxis investigativa en la universidad latinoamericana está mediado por la necesidad de analizar cómo nuestras universidades podrían generar conocimiento a partir de una donde la lógica rígida y mecanicista de hacer ciencia sea desplazada por una propuesta concreta en la que sea la propia trama de la realidad la que señale los métodos de investigación para adentrarnos en ella.

Esto supone, no solo una ruptura en relación con el ser y quehacer investigativo, sino una ruptura epistemológica en tanto se indaga desde lo que Martínez (2009) denomina el cambio en las estructuras lógicas de hacer ciencia, ya que "reta nuestra lógica, reclama una alerta, reclama mayor sensibilidad intelectual, exige una actitud crítica constante" (p.15).

En efecto, si consideramos que la mayor fortaleza del quehacer investigativo es la fragilidad de sus premisas teóricas, en tanto se entiende que los hallazgos son relativos y circunstanciales, entonces, esa actitud crítica y vigilante convendría incorporarla en la praxis para interpretar, explicar y comprender el entorno. Se preguntará probablemente el lector: ¿tendrá sentido tanta versatilidad creativa en términos científicos dada su circunstancialidad? La respuesta es afirmativa porque el acto creativo ha de ser permanente, constante, rítmico y en contradicción continua con el ser (Carvajal, 2005). Ello propicia no sólo la revisión epistemológica y metodológica al interior de las ciencias, sino la configuración de un saber científico, riguroso sí, pero hipotético, por cuanto cada hallazgo científico-teórico representa una respuesta probable a la multidimensionalidad de nuestro contexto.

Se parte entonces de una actitud hermenéutica, en el sentido de la reflexión, del cuestionamiento a las prácticas investigativas universitarias que han propiciado una cultura de lo urgente, de la inmediatez en las respuestas de los universitarios ante las necesidades contextuales. En contraste, lo que se propone es la sustitución de la ortodoxia por una praxis investigativa gestáltica ${ }^{1} \mathrm{y}$ he-

1 Gestalt como estructura "compartida por un grupo humano cuyos elementos no son contenidos concretos, sino concretas condiciones de contenido y del proceso productor de contenidos. Gestalt 
terodoxa, afianzada en procedimientos que emerjan de la singular realidad socio-histórica y de la diversidad y complejidad de los hechos.

Desde esa totalidad, a partir de esa Gestalt se tiene la opción de seguir en la ruta de la fragmentación del saber -obviando que el conocimiento es un hecho histórico- manteniendo la lógica mecanicista dentro de coordenadas teóricas y metodológicas ya establecidas; o por el contrario se tiene la alternativa, como seres sociales, como seres históricos, de interpretar la realidad de acuerdo al contexto en que son ubicadas. Por consiguiente, si el contexto asigna un significado a las cosas, al interactuar cada una de ellas con los elementos del mismo, entonces ¿la realidad puede seguir siendo estudiada de manera fragmentada? ¿Y pasar de largo, ante la premisa de que todas las realidades tienen su significado en una determinada episteme?

En nuestra universidad latinoamericana hemos sido protagonistas de una rémora que ha dejado el paradigma positivista y el método analítico, y es la tendencia a la no reflexión, a la ilusión de la razón y de la objetividad. Como contraparte en otros espacios académicos se procura un conocimiento complejo, sistémico y estructural (Rusque, 2003; Sautu, 2004; Parra, 2008; Martínez, 2009, Moreno 2006, Carvajal, 2005) cuyas consecuencias más significativas son sus propuestas cognitivas y teóricas que intentan comprender y explicar la estructura y la dinámica de los cambios societales y sus rupturas profundas y sistémicas. Es el camino de la heterodoxia en la que se postula la flexibilidad de los

no como estructura antropológica sino, en tal caso, como estructura histórica" (1995, p.52) métodos de investigación, de allí su adaptabilidad a las circunstancias, la apertura a la recreación del método. Desde esa percepción se postula el interrogante clave: ¿qué podemos hacer desde nuestras universidades en la procura de un saber contextual y gestáltico? En este artículo se ha planteado la consecución del siguiente objetivo: proponer estrategias de acción creativas en el quehacer investigativo para el reciclaje de los métodos de investigación y, así, generar una praxis investigativa proactiva, gestáltica y heterodoxa.

\section{Metodología}

Para el logro de ese objetivo se consideró que los métodos son flexibles, complementarios y adaptables a las circunstancias del caso analizado; es decir, se deja una abertura en el camino ante la eventualidad de recrear el método ${ }^{2}$. En este particular la metodología se sustenta en el principio de complementariedad de Niels Bohr (Martínez, 2004, p. 171), destacando la necesidad de no agotar el estudio de la realidad desde una sola perspectiva, punto de vista o enfoque. Se logró la consecución del objetivo de investigación realizando la aproximación al objeto de estudio desde un enfoque creativo, al analizar las representaciones sociales y hacer uso de los métodos y técnicas de acuerdo con el discurrir del propio proceso investigativo.

2 "Lanzarse a investigar hoy, en Ciencias Sociales, sobre todo, después de que en la década de los sesenta se han replanteado en forma crítica todas las bases epistemológicas de los métodos y de la ciencia misma, hace ineludible la reflexión descarnada en torno a los fundamentos que han de soportar todo el trabajo que el investigador se dispone a efectuar [...] la audacia para decidir los propios pasos ha dejado de ser una virtud para imponerse como una exigencia de la propia tarea." (Moreno, 1995, p. 25) 
Se parte, pues, de una postura hermenéuti$\mathrm{ca}^{3}$ para interpretar, la información recolectada por medio de:

- La lectura reflexiva del material hemerográfico y bibliográfico recabado en la investigación documental.

- Las entrevistas semiestructuradas ${ }^{4}$ a informantes clave de tres universidades nacionales y algunas universidades latinoamericanas seleccionadas en la muestra estructural-intencional.

Se realizó un muestreo estructural o relacional, el cual se eligió con base en lo que algún especialista considera que las unidades o elementos en particular harán para responder al objetivo de investigación inmediato (Kainnear \& Taylor, 2003). En consecuencia, se seleccionó una muestra integrada por investigadores de trayectoria reconocida tanto en su país de origen como internacionalmente, y cuya vinculación entre estos sería su adscripción como miembros de redes de investigación en los que ya están planificando el desarrollo de trabajos colaborativos, de tal manera que se eligieron profesores de la Red para la Difusión Científica y Humanística Heterodoxias, (ReDCyH) y de la Red de

3 Una "postura hermenéutica" en la intención de "partir de una realidad concreta, histórico-social, de sus prácticas, lenguajes, discursos, etc.., acceder a sus códigos cognoscitivos y arribar, a través de ellos, a la matriz epistémica que la hace comprensible, a su episteme [...] no puede hablarse, por tanto, de método hermenéutico sino, en tal caso de postura hermenéutica y, en todo caso, metódica, entendida ésta como apertura a toda posibilidad de ejercicios de la acción hermenéutica...no se trata, para mi propósito, de indagar al objeto que hace hablar al sentido sino de escuchar las voces que hablan, que no son objetos, sino vivientes que se comunican en el oído y no por la vista" (Moreno, 2006, pp. 78-80)

4 Se recolectó la información en entrevistas semiestructuradas en la modalidad personal y para los casos de universidades extranjeras (fuera de Venezuela) se realizaron las entrevistas en línea a expertos en el área.
Estudios Organizacionales en América Latina, el Caribe e Iberoamérica (REOALCeI).

La muestra quedó constituida por profesores universitarios que laboran en las siguientes instituciones públicas de educación superior: Universidad Nacional Experimental de los Llanos Ezequiel Zamora (UNELLEZ), La Universidad del Zulia (LUZ), Universidad Centro-Occidental Lisandro Alvarado (UCLA), todas éstas en Venezuela. Y la consulta a informantes clave en universidades latinoamericanas estuvo compuestas por profesores-investigadores de: la Universidad Nacional de Córdoba (UNC) Argentina, Universidad Autónoma de Sinaloa (UAS) y Universidad Estatal de Sonora (UES) en México, Universidad Nacional de Costa Rica (UNA) y Universidad Estatal a Distancia (UNED) de Costa Rica, y la Universidad Técnica de Machala (UTMACH) en Ecuador.

\section{Discusión teórica}

Gestalt y Heterodoxia señalan dos construcciones teóricas que son indicativas de una perspectiva en la que la duda, o por lo menos poner en duda lo que sabe y se piensa es un elemento significativo en la producción del conocimiento. Esta perspectiva implica una acción reflexiva en cuanto a la lectura responsable ${ }^{5}$, más que objetiva, de los resultados de investigación a la luz de la pregunta que guía el proceso de indagación. En este artículo, como la interrogante guía

5 En cuanto a la objetividad, el problema no es la búsqueda de la objetividad, del verdadero sentido de lo que se indaga, más bien el asunto es la responsabilidad, en tanto como investigadores hemos de responder por los resultados de nuestro trabajo. Somos nosotros mismos como investigadores, quienes debemos avalar nuestro hacer. "Pasó el tiempo de la feliz ingenuidad que permitía avanzar por brechas ya abiertas sin mirar hacia atrás" (Moreno, 1995, p25) 
es: ¿qué podemos hacer, como profesores universitarios, desde nuestra praxis investigativa, en procura de un saber contextual y gestáltico? entonces sustentaremos la interpretación en los supuestos teóricos de Gestalt (Perls, 1975, 1977; Robine, 1998, Moreau, 1999; Vazquez-Badin, 2010) y Heterodoxia (Savater y Villena, 1989; Carvajal, 2010a).

¿Para qué teorizar acerca de la heterodoxia? Una de las múltiples respuestas es que el hombre como ser creativo, postula proposiciones contrapuestas a las prevalecientes; en este particular Savater plantea: “Oportet Haereses esse" (Savater y Villena, 1989, p 84). Esto es, la confrontación, la crítica, se refuta no para afianzar un nuevo paradigma pues de ese modo se estaría incurriendo en otra forma de ortodoxia, sino, más bien, para transitar por el camino propio, ese que no ofrece respuesta inmediata y en el que cuestionamos y somos cuestionados.

De manera específica, en cuanto a las ciencias Savater señala:

La ortodoxia por excelencia de nuestro tiempo es la ciencia: en ella se dan las características clásicas de las regiones dogmáticas: lenguaje esotérico, sacerdotes que aguardan el misterio y administran la palabra, convicción de los fieles de que todo tiene explicación y que alguien sabe lo que debe ser sabido respecto a cada cosa, rostro benéfico y rostro destructor de la divinidad, necesidad inexorable y fatal del acontecer, milagros conseguidos tras las debidas rogativas y pagos de diezmos...(Savater y Villena, 1989, p 86)
En la experiencia académica, ¿somos los profesores universitarios esa especie de catedrático-sacerdote que tiene la última palabra? De acuerdo con investigaciones recientes (Carvajal y Melgarejo, 2014; Parra, 2008), en la universidad latinoamericana se presenta un debate, al menos en cuanto a la praxis investigativa en ciencias sociales se refiere, entre una marcada tendencia hacia el conductismo y el cognitivismo (positivismo) por una parte, y por otra, hacia un hacer científico mediado por un paradigma comprensivo y holista (Carvajal, 2010a, p. 121). Esta última tendencia se construye como un modo de acercarse a lo real en el que el significado de investigar está integrado con el compartir, cooperar y cuestionar.

En consecuencia, investigar desde esa última acepción, tal y como lo plantea Moreno se convierte en una pregunta constante "la vida se me convirtió en pregunta, la pregunta en investigación y la investigación en cuestionamiento radical" (1995, p. 15). También se convierte en multiplicidad de respuestas que interpretan una realidad como parte de un mundo intersubjetivo, un mundo común a todos, en el que estamos llamados a la controversia, a no quedarnos en la aparente placidez de la certidumbre, a no anclarnos en la convicción de que lo que se dice, se hace o se piensa, es necesariamente cierto. Es el ejercicio de la refutabilidad popperiana (Popper,1973).

Entonces la heterodoxia aplicada al quehacer científico se sostiene en la actitud crítica y refutable no solo de la teoría, de los constructos teóricos, sino en el modo como interpretamos, comprendemos y explicamos esa realidad social. Aquella realidad en la que individuo y sociedad no son entidades 
separables sino campos mutuamente constituidos, que se relacionan intersubjetivamente en un periodo histórico determinado y soportado por un paradigma ${ }^{6}$ propio de ese período, el cual, con certeza, tiene un origen, un desarrollo y un ocaso en el que es sustituido por otro.

En efecto, el paradigma rige una determinada ciencia durante un período histórico, de tal modo que el cambio se generaría en tanto el investigador mantenga en sus simientes una actitud reflexiva y critica en la praxis, signada por el holismo y la creatividad inmanente de la postura gestáltica ¿Para qué esta actitud gestáltica? En este artículo se postula que el ser histórico practica una realidad concreta, se conoce y es conocido en esa episteme, en una Gestalt. De allí que el constructo tomado del alemán Fritz Perls (1977), Gestalt, es reapropiado desde su clara connotación a la estructura, a la configuración, a la interrelación entre las partes y la relación dinámica entre figura y fondo. Tal y como lo argumenta Martínez, quien propone una especie de actitud gestáltica del investigador en las ciencias humanas "para que un saber no quede despojado de sus propias raíces" (2009, p. 117), es decir no quede fuera de su episteme.

De la teoría Gestalt se toma el constructo de figura y fondo (Perls, 1977, p. 103). Cuando se asume la praxis investigativa desde la

6 "Un paradigma es una imagen básica del objeto de una ciencia. Sirve para definir lo que debe estudiarse, las preguntas que es necesario responder, cómo deben responderse y que reglas es preciso seguir para interpretar las respuestas obtenidas. El paradigma es la unidad más general de consenso dentro de una ciencia y sirve para diferenciar una comunidad científica de otra. Subsume, define e interrelaciona los ejemplares, las teorías y los métodos e instrumentos disponibles." (Ritzer, 1993, p. 598) episteme (fondo) donde se genera, buscamos saber no sólo por qué (explicación), sino indagar en su estructura, enfatizando su totalidad para comprender e interpretar desde esa totalidad, hacia la integración en nuestro foco perceptivo que sería la figura.

Ya no se estarían desfragmentando los elementos constituyentes como partes del fondo (o estructura superior), sino que serían observados como totalidad recíprocamente relacionada. En este enfoque de proceso, la totalidad es vista como un sistema abierto, en el que se enfatiza en lo que está sucediendo, se centra en el "darse cuenta" (Vázquez, 2010, p. 107) de que sujeto y objeto son elementos de una misma episteme que está cambiando constantemente, y en las que figura-fondo son un campo, un sistema con entradas y salidas, orgánicamente regulado.

¿Qué otro aporte significativo se está tomando de la teoría Gestalt que sería aplicable en la propuesta, objetivo, de este artículo? El concepto de "vacío fértil" (Moreau, 1999, pp. 182-183). Estar en un vacío fértil permite ver las estructuras y posibilita trabajar (en términos metodológicos) con lo que va apareciendo, lo que va haciendo figura sobre un fondo, en donde se le da importancia a "lo que es, y no a lo que debe ser" (Perls, 1975, p. 77). Es un estudio de proceso continuo que generaría la acción creativa de quien al observar sabe que no puede forzar los métodos a lo que va apareciendo, en oposición a ese intento fallido de colocar una especie de camisa de fuerza al estudio de una realidad que nos reclama un acto de observación y de comprensión que haría explicito lo implícito, para dar paso a una nueva Gestalt (ver diagrama 1). 
Figura 1. Diagrama número 1

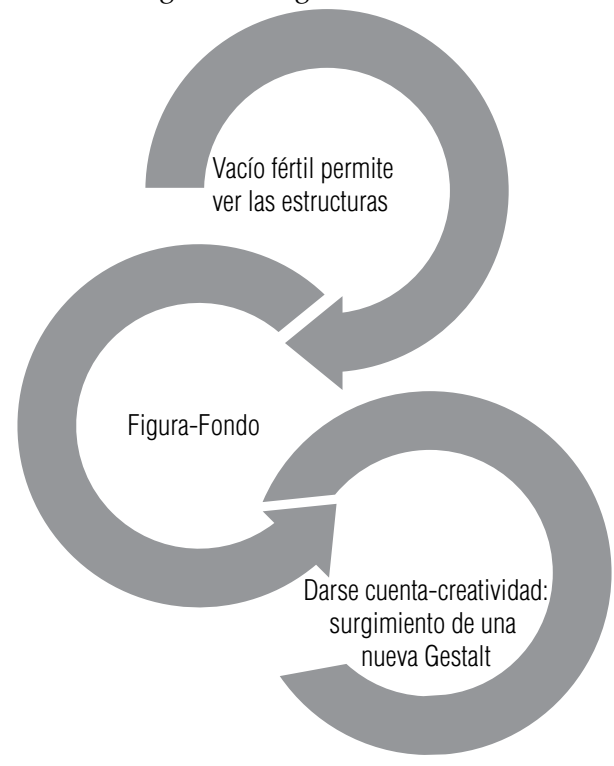

Fuente: elaboración propia, basada en autores citados

\section{Resultados}

Sistema es un constructo absolutamente complejo en el cual tiene su simiente la lectura de los resultados que se presentaran en este aparte. En este sentido, es necesario puntualizar que se ha creado para el análisis de las entrevistas realizadas a los informantes clave una modalidad que reitera la premisa de Bertalanffy (1976, p. 47-49), la cual señala que estamos inmersos en un mundo de sistemas, en el que interactúan entidades complejas, y cuya existencia es interdependiente de los elementos que forman la totalidad.

Aquel principio de interacción recurrente entre los constituyentes de un todo fue el que se hizo evidente al realizar el análisis de las entrevistas a los informantes. De tal modo, se fueron tejiendo hilos y conexiones que dibujaron algo parecido a una constelación de divergencias y convergencias entre los puntos nodales. Distintos actores realizando el análisis de un mismo tema generó la complementariedad de esos enfoques, hasta que finalmente derivaron hacia puntos específicos (ver diagrama 2) en los que las respuestas de los entrevistados convergen en lo que se denominó "significado complejo cultural compartido" (Carvajal. 2010a, pp. 122-123). Es decir: el significado que han dado los propios informantes a un constructo formado desde la acción social, y vivenciados en su experiencia concreta de la praxis investigativa.

Desde una postura hermenéutica se descubren esos significados, conservando su singularidad en el contexto del cual forman parte, y se establece un proceso de reconstrucción de sentidos del discurso, en el que se atiende la intención de traspasar el sentido superficial, e indagar en la polisemia, en los múltiples significados. Allí se reconoce que el significado del texto va a ser "leído" con el influjo de nuestra condición de seres históricos, con nuestro modo de ver, con nuestras actitudes y conceptos ligados a la lengua y los estilos de vida; ${ }^{7}$ un horizonte de experiencias, creencias, normas, mapas mentales, que pertenecen a un contexto histórico propio. Se hizo así un acto de observación mediado por ese horizonte.

7 "Un ser humano descubre su finitud en el hecho de que, ante todo, se encuentra a sí mismo dentro de una tradición o tradiciones. Y como la historia me precede a mí y a mi reflexión, como yo pertenezco a la historia antes de pertenecerme a mí mismo, el prejuicio también precede al juicio y la sumisión a una tradición precede a su examen. El régimen de la conciencia histórica es el de una conciencia expuesta a los efectos de la historia. Si, por tanto, no podemos arrancarnos a nosotros mismos de nuestra pertenencia a la historia o situarnos a nosotros mismos a tal distancia de ella que el pasado se convierta en un objeto para nosotros, entonces tenemos que confesar que siempre estamos situados dentro de la historia de tal suerte que nuestra conciencia es libre para enfrentarse cara a cara con el pasado por medio de un acto de soberana independencia. Se trata entonces, más bien, de tornarnos consientes de la acción que nos afecta y de aceptar que el pasado, que es una parte de nuestra propia experiencia, nos impide hacernos cargo totalmente de él, de aceptar en cierto modo su verdad" (Ricoeur, 1973: 157) 
Figura 2.

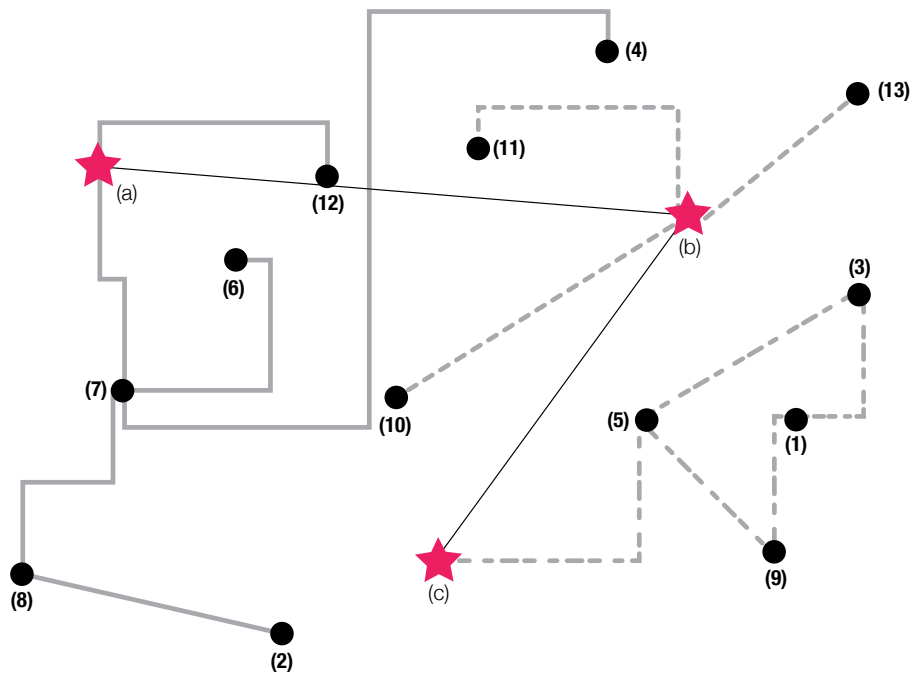

\section{Leyenda de Puntos:}

- $\quad$ Apropiación social del conocimiento (1)

- Interdisciplinariedad (2)

- Políticas de investigación sustentables (3)

- Trabajo colaborativo (4)

- Presupuestos universitarios para la investigación (5)

- Quehacer investigativo en estancos cerrados (6)

- $\quad$ Articulación de docencia e investigación (7)

- Competencias del investigador (8)

- Cultura investigativa reguladora y ortodoxa (9)

- Mecanismos de divulgación y difusión de resultados de investigación (10)

- Requerimientos societales (11)

- $\quad$ Creatividad e innovación (12)

- Gestión y acción investigativa (13)

- Señala la intersección entre puntos nodales

En consecuencia y tomando en consideración las dos premisas básicas de las que parte este artículo:

- Nuestra mente observa e interpreta la realidad en movimiento fluyente, es decir se va del todo a las partes, de la figura al fondo, en movimiento dialéctico; develando una realidad que sólo es conocida en los límites concretos de nuestra percepción (Carvajal, 2010b, p. 456).
- Las universidades están llamadas a ser un espacio en el que se fomente el cambio continuo, por medio de su quehacer docente, investigativo y de extensión (Carvajal, 2001, p. 31),

A continuación, se pasará a dar cuenta al lector de los resultados obtenidos.

Primero se agruparon las respuestas en tres puntos de interpretación, que se dibujaron 
como constelaciones (presentados en el diagrama 2), en las que estarían interactuando los significados complejos culturales compartidos (S3C) identificados en la narrativa de los informantes. Se asociaron en tres puntos nodales, estos son: (a) Fuerzas y falencias en la praxis investigativa, (b) Investigación y entorno, y (c) Gestión para la investigación, y son presentados a continuación en el siguiente cuadro (ver Cuadro 1).

Tabla 1.

Puntos nodales que hacen figura en la narrativa de los informantes en su experiencia concreta de la praxis investigativa universitaria

\begin{tabular}{|c|c|c|c|}
\hline País & $\begin{array}{l}\text { Fuerzas/Falencias } \\
\text { en la praxis investigativa }\end{array}$ & Investigación y entorno & $\begin{array}{l}\text { Gestión para la } \\
\text { investigación }\end{array}$ \\
\hline $\begin{array}{l}\text { Argentina } \\
\text { (UNC) }\end{array}$ & $\begin{array}{l}\text { Interdisciplinariedad (2). La } \\
\text { praxis investigativa inter- } \\
\text { disciplinaria se declara, en } \\
\text { términos de línea de acción } \\
\text { necesaria, más no se tradu- } \\
\text { ce en acciones concretas. } \\
\text { Sin embargo, en los niveles } \\
\text { de postgrado se han consti- } \\
\text { tuido unidades de gestión } \\
\text { en la que es necesaria } \\
\text { la interdisciplinariedad, } \\
\text { entonces se favorece el } \\
\text { desarrollo de programas y } \\
\text { proyectos en los que parti- } \\
\text { cipan distintas facultades. } \\
\text { Y esto también auspicia la } \\
\text { articulación docencia-inves- } \\
\text { tigación (7). Los estudiantes } \\
\text { que han participado en } \\
\text { tesis y proyectos de inves- } \\
\text { tigación, han contribuido, } \\
\text { junto con sus docentes, en } \\
\text { la aplicación de enfoques } \\
\text { vanguardistas que generan } \\
\text { apertura en la forma de } \\
\text { hacer ciencia, sobretodo en } \\
\text { el área de metodología en } \\
\text { Ciencias Sociales. }\end{array}$ & $\begin{array}{l}\text { Gestión y acción de la } \\
\text { investigación (13). La uni- } \\
\text { versidad pública ha sido } \\
\text { beneficiada por políticas } \\
\text { decisivas para impulsar } \\
\text { la investigación y el desa- } \\
\text { rrollo. En este contexto, se } \\
\text { han adelantado diversos } \\
\text { programas que favorecen } \\
\text { la investigación básica y } \\
\text { aplicada y, especialmente, } \\
\text { el trabajo interdiscipli- } \\
\text { nario y la difusión del } \\
\text { conocimiento por medio } \\
\text { de publicaciones científicas y } \\
\text { de divulgación (10). }\end{array}$ & $\begin{array}{l}\text { Apropiación social del cono- } \\
\text { cimiento (1). Se han dado } \\
\text { pasos importantes que } \\
\text { propician una comunica- } \\
\text { ción más horizontal entre la } \\
\text { universidad que genera el } \\
\text { conocimiento y la sociedad } \\
\text { que le asigna tal función, y } \\
\text { le demanda respuestas a } \\
\text { los problemas que acucian } \\
\text { a la propia sociedad. } \\
\text { Políticas de investigación } \\
\text { sustentables (3). Se han } \\
\text { suscrito convenios para } \\
\text { apoyar programas de vin- } \\
\text { culación institucional y así } \\
\text { favorecer la realización de } \\
\text { actividades conjuntas, y el } \\
\text { intercambio de estudiantes, } \\
\text { docentes e investigadores }\end{array}$ \\
\hline
\end{tabular}




\begin{tabular}{|c|c|c|c|}
\hline País & $\begin{array}{l}\text { Fuerzas/Falencias } \\
\text { en la praxis investigativa }\end{array}$ & Investigación y entorno & $\begin{array}{l}\text { Gestión para la } \\
\text { investigación }\end{array}$ \\
\hline $\begin{array}{l}\text { Costa Rica } \\
\text { (UNED } \\
\text { y UNA) }\end{array}$ & $\begin{array}{l}\text { Interdisciplinariedad (2). Se } \\
\text { hacen intentos, individua- } \\
\text { les o de grupos de investi- } \\
\text { gación, para generar una } \\
\text { relación fuerte entre lo que } \\
\text { se produce en la actividad } \\
\text { investigativa y lo que se } \\
\text { enseña. Pero la mayor falla } \\
\text { es el desarrollo de una pra- } \\
\text { xis investigativa en estancos } \\
\text { cerrados, la investigación } \\
\text { se ha dedicado, en mayor } \\
\text { medida, a las descripciones } \\
\text { técnicas, y poco se hace } \\
\text { desde el punto de vista de } \\
\text { la interpretación holística, } \\
\text { interdisciplinaria. } \\
\text { Competencias del investiga- } \\
\text { dor (8). Se ha planteado } \\
\text { en el nivel curricular un } \\
\text { perfil por competencias del } \\
\text { investigador que incluye: } \\
\text { liderazgo, articulación, } \\
\text { emprendedurismo y } \\
\text { creatividad. } \\
\text { Falencias: a pesar de las in- } \\
\text { tención de realizar un traba- } \\
\text { jo colaborativo (4) se continua } \\
\text { observando un quehacer en } \\
\text { estancos cerrados (6). }\end{array}$ & $\begin{array}{l}\text { Gestión y acción investigativa } \\
\text { (13). Se promueven desde } \\
\text { la gerencia universitaria la } \\
\text { investigación intercátedra } \\
\text { y con la dotación de fondos } \\
\text { se estimula la participación } \\
\text { en proyectos interuni- } \\
\text { versitarios nacionales, La } \\
\text { debilidad, está en la parti- } \\
\text { cipación en red en el nivel } \\
\text { internacional. } \\
\text { Se están realizando } \\
\text { esfuerzos para que la in- } \\
\text { vestigación tenga impacto } \\
\text { regional; y se atiendan los } \\
\text { requerimientos de la sociedad } \\
\text { (11); sin embargo, los } \\
\text { centros e institutos están } \\
\text { centralizados en la capital. } \\
\text { Entonces las necesidades } \\
\text { del entorno son atendidas } \\
\text { desde el punto de vista de } \\
\text { las demandas del mercado } \\
\text { de trabajo, específicamente } \\
\text { en generar cohortes y más } \\
\text { cohortes de graduandos. }\end{array}$ & $\begin{array}{l}\text { Políticas de investigación } \\
\text { sustentable y presupuesto ( } 3 \\
\text { y 5). No se puede tener una } \\
\text { política de investigación } \\
\text { sustentable en el tiempo, } \\
\text { cuando en materia de } \\
\text { presupuestos no se le da } \\
\text { prioridad. Parece que la } \\
\text { idea de graduar profesiona- } \\
\text { les de manera rápida y en } \\
\text { una mal entendida compe- } \\
\text { titividad para el mercado } \\
\text { laboral, ha concentrado } \\
\text { el quehacer universitario } \\
\text { a la docencia, de manera } \\
\text { desvinculada de la investi- } \\
\text { gación y la extensión. }\end{array}$ \\
\hline $\begin{array}{l}\text { Ecuador } \\
\text { (UTMACH) }\end{array}$ & $\begin{array}{l}\text { En Ecuador, pese al mo- } \\
\text { vimiento de renovación } \\
\text { de la educación superior, } \\
\text { impulsado por el actual } \\
\text { gobierno, las actividades } \\
\text { de investigación en las que } \\
\text { debería darse la innovación } \\
\text { y creatividad (12) se realizan } \\
\text { en estancos cerrados (6), y } \\
\text { con escasa posibilidad de } \\
\text { desarrollo de pesquisas } \\
\text { interdisciplinarias (2) porque } \\
\text { el docente es sobrecargado } \\
\text { con trámites administra- } \\
\text { tivos para la acreditación } \\
\text { o recategorización de la } \\
\text { universidad (UTMACH) } \\
\text { Prevalece la burocracia y } \\
\text { la evaluación y control del } \\
\text { "paso" dado. La investiga- } \\
\text { ción apegada a la norma. }\end{array}$ & $\begin{array}{l}\text { Requerimientos societales } \\
\text { (11). Se desea alcanzar una } \\
\text { transformación a nivel de } \\
\text { la institución considerando } \\
\text { realidades ajenas al entorno } \\
\text { nacional. Todo esto con- } \\
\text { lleva que la investigación } \\
\text { y la formación profesional } \\
\text { se enmarque en realidades } \\
\text { ficticias, no respondien- } \\
\text { do a las necesidades del } \\
\text { entorno socio-productivo. } \\
\text { Pareciera que la gestión y } \\
\text { acción investigativa (13) se } \\
\text { asentara en la dinámica de } \\
\text { la tecnociencia reactiva y } \\
\text { no a criterios académicos } \\
\text { sociales y humanistas. }\end{array}$ & $\begin{array}{l}\text { Cultura investigativa } \\
\text { reguladora y ortodoxa (9). La } \\
\text { cultura investigativa tiende } \\
\text { a ser reguladora y ortodoxa, } \\
\text { se genera una distorsión } \\
\text { de la actividad científi- } \\
\text { ca debido a exceso de } \\
\text { controles y normas desde } \\
\text { la gerencia central. De allí } \\
\text { que las políticas de investiga- } \\
\text { ción (3), lejos de estimular } \\
\text { la innovación, crean un } \\
\text { efecto adverso como lo es } \\
\text { la repetición y consecuente } \\
\text { desvalorización de la acti- } \\
\text { vidad científica. Presupues- } \\
\text { tos universitarios para la } \\
\text { investigación (5). }\end{array}$ \\
\hline
\end{tabular}




\begin{tabular}{ll}
\hline País & $\begin{array}{l}\text { Fuerzas/Falencias } \\
\text { en la praxis investigativa }\end{array}$ \\
\hline \hline México & Creatividad e innovación (12) \\
yAS y UES) & México, la praxis inves- \\
& tigativa se impulsa por \\
& medio de los lineamientos \\
& que dictan las instancias \\
& federales como el CONA- \\
& CYT (Consejo Nacional de \\
& Ciencia y Tecnología) y el \\
& PRODEP (Programa para \\
& el Desarrollo Profesional \\
& Docente), entonces las ins- \\
& tituciones deben apegarse \\
& a estos lineamientos. Ahora \\
& el resultado es que se
\end{tabular}
genera más competencia y menos trabajo colaborativo, imperando una situación de desigualdad. Se va generando una hegemonía en la creación del conocimiento (un pequeño número de instituciones bien posicionadas en los rankings mundiales, cuyos investigadores son los que se destacan por sus trabajos de investigación).

Hace falta fortalecer la interdisciplinariedad (2), y en los recientes 10 años, los cuerpos académicos y grupos de investigación se han integrado con investigadores y cuerpos académicos de otras instituciones (tanto nacionales como internacionales) de distintas disciplinas, pero con las cuales se comparten objetivos comunes.

\section{Investigación y entorno}

Gestión y acción investi-

gativa (13). Actualmente, el tiempo y el apoyo para realizar en la institución (UES), el quehacer investigativo es muy limitado. Se observa poco estímulo para motivar al docente a realizar investigación y no existen condiciones para el desarrollo de ésta. La política es centrarse más en la docencia y dejar en segundo término el aspecto de la investigación. Si bien en años anteriores hubo esfuerzos por impulsar e incentivar la investigación, en este momento para la institución (UES) no es una actividad que tenga prioridad. Difícilmente se puede atender los requerimientos societales (11).

Es poco lo que se está haciendo, los directivos siguen preocupados por resolver lo urgente pero no lo importante. En este sentido, los requerimientos sociales son atendidos en el incremento de la matrícula, pero se está contraponiendo con la calidad $\mathrm{y}$, peor aún, con el desarrollo de programas de investigación de cara a los urgentes problemas locales y nacionales, como el hambre y la sustentabilidad ecológica.

\section{Gestión para la investigación}

Politicas de investigación (3) y presupuesto (5). Existe un retardo en la asignación de recursos para la investigación, porque estos no son asignados directamente por la universidad, sino que están centralizados en la Secretaría de Educación Superior de México. Entonces se genera una cultura investigativa (9) que se hace cada vez más ortodoxa y regulada por los cánones y exigencias del gobierno central. Además, no hay espacio para que el investigador trabaje en los proyectos, porque tiene una carga académica y de gestión que consume toda su jornada laboral. Si bien en años anteriores hubo esfuerzos, desde las universidades públicas, por impulsar e incentivar la investigación, en este momento no es una actividad que tenga prioridad y los docentes que hacen investigación es porque, por lo general, lo hacen fuera del marco institucional. 


\begin{tabular}{|c|c|}
\hline País & $\begin{array}{l}\text { Fuerzas/Falencias } \\
\text { en la praxis investigativa }\end{array}$ \\
\hline $\begin{array}{l}\text { Venezuela } \\
\text { (UNELLEZ, LUZ } \\
\text { y UCLA) }\end{array}$ & $\begin{array}{l}\text { Quehacer investigativo en } \\
\text { estancos cerrados (6) y Creati- } \\
\text { vidad e innovación (7). Poco } \\
\text { poder innovador o transfor } \\
\text { mador tanto hacia el en- } \\
\text { torno externo (pertinencia } \\
\text { social), como en la propia } \\
\text { práctica investigativa que } \\
\text { se desarrolla en estancos } \\
\text { cerrados. }\end{array}$ \\
\hline
\end{tabular}

No obstante, como punto a favor: se sigue, a pesar de la crisis económica y social del país, generando investigaciones en algunos campos de las ciencias en las que los profesores buscan alianzas interuniversitarias en el nivel nacional e internacional, lo cual demanda gran capacidad creativa e innovadora por parte de los involucrados.

El otro punto es que se siguen abriendo espacios en Venezuela, hacia la interdisciplinariedad (2) la cual posibilita evaluar fenómenos que involucran la percepción desde distintas perspectivas, y al parecer es la propia crisis socioeconómica y política la que genera una fuerza que estimula las competencias de liderazgo $y$ trabajo colaborativo entre los docentes (8).

\section{Investigación y entorno $\quad \begin{aligned} & \text { Gestión para la } \\ & \text { investigación }\end{aligned}$}

Gestión y acción investiga-

tiva (12) y Requerimientos

societales (11). La gestión

investigativa tanto en la

UNELLEZ; como en UCLA

y LUZ está afectada por

una política del gobierno

central de escaso estí-

mulo a la investigación y

desarrollo. Entonces los

requerimientos societales (11)

son atendidos mediante

convenios particulares de grupos de investigación especializados que obtienen financiamiento de empresas privadas $\mathrm{u}$ organismo bilaterales. Y en algunos casos con financiamiento del mismo docente, no obstante, esta modalidad ya no es posible por el déficit en los sueldos de los profesores universitarios.

Mecanismos de divulgación (10). La divulgación de los resultados de investigación se ve limitada, porque no hay presupuesto asignado para asistencia a eventos científicos, el único mecanismo que le ha quedado a los investigadores es la publicación en revistas indexadas, tanto nacionales, como internacionales. Solo que, en el caso de las publicaciones nacionales, en la actualidad se restringen a las revistas digitales, ya que no hay papel para cubrir la demanda de revistas impresas.
Políticas de investigación (3), Cultura investigativa reguladora y ortodoxa (5). Hay una clara tendencia, que comenzó a inicios de este siglo, hacia la precarización de la investigación, inicialmente en universidades intervenidas por el gobierno central como la UNELLEZ, y que luego se hizo extensivo a las universidades autónomas (como la LUZ y UCLA) Entonces el quehacer investigativo se va reduciendo a una acción obligante de ciertas prácticas que apuntan más a la dinámica socio comunitaria que a la producción intelectual.

Presupuesto (5). En general el presupuesto asignado a las universidades es insuficiente $^{8}$, y por consiguiente el correspondiente a la actividad de investigación es prácticamente nulo. De tal manera que es tarea titánica, de parte del personal docente, poder realizar una investigación, así sea de bajo costo.

Fuente: interpretación de entrevistas realizadas a informantes clave.

8 La Asociación Venezolana de Rectores Universitarios (AVERU, 2016) señala: el presupuesto asignado a las Universidades venezolanas para su funcionamiento y el pago de sueldos y salarios resulta inferior a sus necesidades reales, lo que afecta el nivel de vida de los miembros de la comunidad universitaria, y las condiciones en que se desarrollan las actividades académicas, de investigación, extensión, administrativas y estudiantiles. 
En el análisis se puede observar que, a pesar de la heterogeneidad de la muestra en cuanto a las diferencias geográficas y del contexto histórico social, los investigadores aluden a elementos que están presentes en todos los países, y que configuran algunas similitudes en cuanto a qué factores son los que caracterizan su quehacer investigativo. En el caso de Venezuela, sí se aprecia una diferencia acentuada en cuanto a la percepción de ese elemento que hace figura, porque la propia situación socioeconómica, marcada por la inestabilidad política y crisis humanitaria $^{9,10}$, repercute en todos los ámbitos de acción del ciudadano venezolano.

Una de las interrogantes que se le formularon a los entrevistados era: "Hace más de 20 años la UNESCO (1998) viene insistiendo en que los países en desarrollo sólo lo alcanzarán,

9 "Estoy muy preocupado por la situación actual, en la que las necesidades básicas no pueden ser cubiertas, como la comida, el agua, la sanidad, la ropa, no están disponibles. Eso crea una crisis humanitaria en Venezuela -dijo el secretario general-. Toda esta situación está creada por la inestabilidad política. Y antes que nada tiene que haber estabilidad política". (Ban-Ki-moon, http://www.el-nacional.com/GDA/ Ban-Ki-moon-Venezuela-crisis-humanitaria_0_901111941.html

10 Según los datos del Banco Central de Venezuela (Índice Nacional de Precios al Consumidor, disponible en http://www.bcv.org.ve/) lo que se observa es que durante el período 2008-2014 se octuplicó el costo de la vida. Siendo la base de 100 en 2007, en 2014 el índice alcanza los 800. En otros términos, cada vez el dinero vale menos, no hay recuperación del poder adquisitivo de la población en general. Y si bien en ningún año se llegó a una inflación anual de más de 100 o rondando los 80, en todos los casos está por encima de los dos dígitos y es superior a $20 \%$. Y cuando se habla de inflación promedio superior a los dos dígitos es porque existe una caída significativa del poder adquisitivo en la población, del poder de compra. Ver también cifras actualizadas del Instituto Nacional de Estadísticas http://www. ine.gov.ve/index de las que se colige: que el INPC registró hasta septiembre del año 2015 una variación acumulada de 108,7\%. A esto se agrega el análisis realizado por el economista venezolano Francisco Faraco http://informe21.com/economia/ quien afirma que el Banco Central de Venezuela está obligado a tomar los precios oficiales, que no son los precios reales del mercado. Entonces hay un desfase entre lo que el BCV publica y lo que el consumidor aprecia, por lo que calcula en 300 por ciento la tasa real de inflación en el país al cierre de 2015 . con una cualificada y competente preparación de sus profesionales universitarios; es decir: la generación de conocimiento como única fuente de ventaja competitiva sostenible, para la solución de los problemas más elementales que nuestro contexto social, económico, ambiental y cultural nos demanda. ¿Desde su perspectiva, ese llamado ha sido atendido en la praxis investigativa universitaria que se realiza en la institución donde labora?" Y en función de las respuestas dadas, se llegó al siguiente horizonte hermenéutico: la universidad latinoamericana ha tenido que atender multiplicidad de demandas que han superado su capacidad de respuesta, y esas demandas contextuales van desde lo urgente: desastres naturales y provocados, hasta lo cotidiano: enfermedades recurrentes, seguridad alimentaria, indicadores socio-económicos, pasando por riesgos de rupturas y cambios profundos que se expresan en los ámbitos políticos y sociales.

El entorno exige a las universidades la disposición de acompañar los cambios desde su praxis investigativa (Carvajal, 2008), no obstante, este compromiso no puede ser asumido con determinación si el universitario no encara dos retos: a) el cambio en cuanto su ser y que hacer investigativo $y, b$ ) el reciclaje de sus métodos de investigación como estrategias de anticipación y acción en el quehacer investigativo universitario.

En cuanto al primer reto, a continuación se presentan, en el Cuadro 2, las propuestas realizadas por los informantes en el que se visibiliza la relación de aportes dados por los entrevistados desagregada por países. Después pasaremos, en función del diagnóstico realizado y las opciones para el cambio del quehacer investigativo, a dar 
respuesta a la pregunta clave en este artículo: ¿qué podemos hacer desde nuestras universidades en la procura de un saber contextual y gestáltico? De ahí, se presentarán las estrategias para el reciclaje de los métodos de investigación como mecanismos de acción creativos en el quehacer investigativo universitario.

Tabla 2

Opciones para el cambio, realizadas por los informantes en cuanto al que hacer investigativo universitario

\section{PAÍS}

Propuestas para el cambio

Argentina Gestión y acción investigativa (13)

(UNC)

La universidad latinoamericana podrá dar respuestas al entorno cambiante en la medida en que sus políticas en la gestión y acción investigativa estén orientadas con políticas de desarrollo sustentable que desafíen el tradicional esquema de dependencia de los países centrales. Políticas de independencia científico-tecnológica, producción diversificada, con la consecuente distribución equitativa de la riqueza, son algunas de las acciones a las que la universidad debe contribuir. Además, en cuanto al Trabajo colaborativo (4) es necesario que las universidades latinoamericanas suscriban programas de vinculación institucional que favorezcan la realización de actividades conjuntas, y el intercambio de estudiantes, docentes e investigadores, y que esos acuerdos se hagan dentro de una cultura investigativa proactiva.

Costa rica Quehacer investigativo en estancos cerrados (6) y Creatividad e innovación (7)

(UNED Y UNA) Un factor clave para el cambio del quehacer investigativo hacia la proactividad es en la formación del investigador. Un cambio del perfil por competencias en el cual se consideran elementos como liderazgo, articulación, emprendedurismo, creatividad como elementos transversales en programas permanentes de formación en capacidades en investigación (tanto para docentes, como para estudiantes).

La indicación principal en cuanto a Políticas de investigación sustentable se refiere (3) sería el fortalecimiento de las alianzas con los actores sociales: ONG, gobiernos locales y algunas empresas que tengan presencia y liderazgo en las regiones del país de forma tal que no solo sea posible complementar el presupuesto (5) asignado por el gobierno central, sino en sus consecuencias inmediatas para las actividades de investigación y transferencia, haciéndolas más efectivas y con mayor incidencia en el entorno socio-productivo, de forma tal que la investigación generada en las universidades se constituya en promotora de cambio social.

\begin{tabular}{ll}
\hline Ecuador & Para que la universidad atienda los requerimientos societales (11), desde la creatividad \\
(UTMACH) & e innovación (12) es necesario que la Gestión y acción investigativa (13) mediada por la \\
& Senescyt (Secretaría Nacional de Educación Superior Ciencias y Tecnología) no solo \\
& centre el apoyo en la tecnociencia, sino en las ciencias sociales y humanas, de tal mane- \\
& ra que la universidad se involucre y comprometa con la actividad de investigación con \\
& la finalidad de generar cambios palpables y enfocados a resolver y atender problemas \\
& sociales y económicos en correspondencia con la sociedad civil.
\end{tabular}

Además, se hace necesario acompañar los cambios con una formación académica de alta calidad para los docentes [Competencias del investigador (8)], quienes están rezagados en comparación con sus pares de la región, entonces se plantea la creación de intercambios científicos y de investigación con otras universidades y centros de investigación, tanto a nivel nacional e internacional, de tal forma que propicie la generación de proyectos de participación conjunta con otros profesores investigadores y que puedan responder a diferentes interrogantes de lo que actualmente acontece en los países que se involucren. 


\section{PAÍS}

\section{Propuestas para el cambio}

México La propuesta que formulan los docentes-investigadores de la UAS y la UES se centran en cuatro aspectos que hacen interacción: Interdisciplinariedad (2) Competencias del investigador (8), y Mecanismos de divulgación y difusión de resultados de investigación (10). En este sentido exhortan a que se siga promoviendo la habilitación académica (maestrías y doctorados) de los profesores en instituciones élite de calidad, así como la constitución de redes académicas tanto nacionales como internacionales que ayuden a que investigadores de instituciones de prestigio trabajen con sus pares de otras instituciones.

Desarrollo constante del trabajo inter y multidisciplinario, y llevarlo a otros ámbitos, es decir, trabajar en conjunto con otros Centros de Investigación en el nivel nacional e internacional para intercambiar experiencias de investigación y realizar análisis en contextos diferentes y poder proponer y considerar soluciones a problemáticas que parecen, ser similares en muchos países, donde indicadores sociales, económicos, políticos, de género, de responsabilidad social y transversalidad es el común denominador para ellos.

Desde la perspectiva de las competencias del investigador, es necesario revisar aspectos pedagógicos y disciplinarios en las mallas curriculares de los diferentes programas académicos que se imparten. Es importante incorporar temáticas acordes a los contextos y situaciones actuales del entorno económico, social, legal, ambiental, político, tecnológico y humanístico, de tal manera que los estudiantes, profesores y autoridades se involucren en un proceso transformador hacia la generación de políticas públicas que apoyen la actividad y praxis investigativa y que, por ende, respondan y contribuyan a solucionar y atender problemáticas identificadas por la sociedad y el mismo entorno universitario.

Venezuela

(UNELLEZ,

Cualquier cambio en el campo de la acción investigativa debe considerar algunas

LUZ y UCLA) orientaciones generales entre las cuales se podrían indicar:

Articulación de la docencia y la investigación (7) La revalorizar la investigación como el mecanismo que alimenta la docencia pertinente y de calidad, no se puede exigir una formación adecuada de los estudiantes si los profesores se convierten en simples "dadores de clases" sin mayor incentivo para dedicarse a la investigación.

Para construir, fomentar y mantener una cultura investigativa (9), no ortodoxa, es necesario la reivindicación del trabajo docente, no sólo desde el punto de vista salarial, que es fundamental en este momento en Venezuela, sino desde el plano del respeto al espacio ya ganado por muchos investigadores reconocidos nacional e internacionalmente. Y qué pese a ello están obligados a laborar en espacios donde la cultura de la obediencia es la que prevalece en la gestión investigativa. Es fundamental hacer prevalecer el ejercicio intelectual por encima del político.

Fuente: interpretación de entrevistas realizadas a informantes clave. 
ESTRATEgIAS PARA EL RECICLAJE DE LOS MÉTODOS DE INVESTIGACIÓN Y EL CAMBIO EN LA PRAXIS INVESTIGATIVA UNIVERSITARIA

De los Cuadros 1 y 2 se desprende que los investigadores entrevistados se mueven en un estado de tensión entre "su quehacer investigativo" y "el hacer investigación en un contexto de incertidumbres", interactúan del desorden al orden y de nuevo al desorden, en un ciclo de experiencias que, de acuerdo con los cambios del entorno, le obliga a una mayor capacidad de respuesta y flexibilidad. Entonces, sus estrategias de acción no sólo tendrán que adaptarse al desarrollo del entorno sino anticiparse porque, a fin de cuentas, el ser humano no tiene un modo único y estático de observación, al contrario, el proceso de pensar y discernir acerca de su propia realidad le imposta la necesidad de ser gestáltico en la comprensión e interpretación de los hechos sociales y naturales. En consecuencia, se han de dar nuevas replicas a un contexto cambiante que les exige a nuestras universidades respuestas enmarcadas en los requerimientos sociales, económicos y ecológicos, de una población diversa, y que reclama su sustentabilidad.

Desde la anterior consideración, tomando en cuenta los significados complejos culturales compartidos (S3C) y las interacciones recurrentes entre estos, que fueron agrupadas como puntos nodales (fuerzas/falencias en la praxis investigativa, investigación y entorno, y gestión para la investigación), a continuación se presentan las estrategias para la acción orientadas al reciclaje de los métodos de investigación para propiciar el cambio proactivo en el quehacer investigativo de las universidades latinoamericanas.

\section{Estrategias PARA LA ACCIÓN}

1. Cambiar la articulación docencia e investigación (7). Para que este cambio no sea solo un acto declarativo, es imprescindible que el personal docente asuma, desde su propia iniciativa, su cuota parte de responsabilidad en innovar y competir, no solo porque es una política a seguir. No ha de verse como un mandato al cual se responde de manera reactiva y ortodoxa; se trata, más bien, de forjar un compromiso de cooperación para co-crear y compartir conocimiento entre pares, hacia el contexto histórico que lo demanda.

2. Crear e innovar (12). La heterodoxia aplicada al quehacer científico se sustenta en la necesaria actitud crítica y el ejercicio de refutabilidad en el modo como interpretamos, comprendemos y explicamos el contexto. En este particular, el docente-investigador además del dominio pedagógico para el diseño y estructuración de materiales y contenidos programáticos, requerirá de alta capacidad para generar investigación innovadora y conocimiento actualizado, al menos, en sus áreas de enseñanza-aprendizaje. La innovación asociada a la controversia, no para disentir de modo especulativo, sino para generar un pensamiento irradiante que de apertura a nuevos conocimientos y a nuevas prácticas investigativas de acuerdo con el sujeto-objeto de estudio. 
3. Recrear permanentemente las competencias del investigador (8), El concepto de "vacío fértil" (Moreau, 1999), tomado de la teoría Gestalt, posibilita estudiar de la totalidad lo que va emergiendo, lo que va haciendo figura sobre un fondo. De tal manera que quien interpreta la realidad ha de contar con aptitudes que promuevan el ejercicio investigativo proactivo, divergente y reflexivo, lo que implica, comparar, clasificar, analizar, interpretar y sintetizar las diferentes experiencias investigativas, para dar paso la investigación responsable, desde la ética del compromiso consigo mismo y con el entorno.

4. Impulsar el conocimiento transversal, tanto en la investigación como en la docencia (7). Es necesario planificar y ejecutar acciones concretas para desarmar los estancos cerrados (6) que limitan la construcción del conocimiento. El concepto de transversalidad debe entenderse como acción educativa que se sostiene en la integración de lo diferente, en la construcción de nuevos objetos de conocimiento, en la reflexión sobre el otro y la totalidad, para crear nuevas formas de abordajes de un mismo tema, el cual es analizado desde diferentes perspectivas disciplinarias y usando distintos métodos de estudio. En esta visión sistémica no es posible fragmentar las partes y estudiarlas fuera de contexto. Se entiende que los elementos constituyentes son partes del fondo y son observados como totalidad recíprocamente relacionada.

5. Consolidar una cultura organizacional flexible: para erradicar la cultura investigativa reguladora y ortodoxa (9) y generar políticas de investigación sustentable (3) es necesario pasar de un modelo basado en la acumulación de conocimiento a otro fundamentado en una actitud activa y permanente de contacto y reconocimiento del entorno. Es necesario generar capacidades desde la propia organización universitaria para adaptarse con agilidad y eficiencia a los cambios y a las demandas de la sociedad formando para lo desconocido. Con una propuesta de educación permanente se crean oportunidades de actualización y adaptación a las realidades cambiantes y poco previsibles. Se estaría propiciando una modalidad de investigación proactiva, y además gestáltica en tanto el quehacer investigativo está fundado en la aprehensión de la figura y fondo, y no sólo de una parte de esa totalidad.

6. Atender a los requerimientos societales (11), para esto es necesario la cooperación entorno laboral-universidades, asumida como modalidad educativa, en la que el empleador y la universidad comparten la responsabilidad social y económica en la formación del investigador (tanto docente, como estudiante). Así se transfiere conocimiento de la universidad hacia el entorno socio-productivo y se transfieren problemas y aprendizajes prácticos desde ese entorno hacia la universidad. En un ciclo de experiencia que se va recreando en el trabajo colaborativo y en la aceptación de nuevos retos para fortalecer, al mismo tiempo, el presupuesto universitario para la investigación (5).

7. Practicar el trabajo colaborativo (4) y la interdisciplinariedad (2). Es claro que no necesariamente el trabajo en colaboración 
entre pares se hace desde la interdisciplinariedad. No obstante, para el desarrollo de una praxis investigativa interdisciplinaria sí es necesaria la colaboración que puede estar estructurada en redes académicas, que estarían accionando desde principios de complementariedad, competencia, transferencia de conocimiento y experiencias. Estas redes serían estimuladas desde una gestión y acción investigativa (13) proactiva. Y la integración de redes universitarias interregionales sería una vía para racionalizar los recursos económicos provenientes del Estado, para fortalecer la función académica, científica y social de la región y en cada país. Se propone, entonces, una cultura investigativa fundada en el trabajo en equipos con alto grado de autonomía y libertad creativa. En donde en las redes de comunicación es fundamental el despliegue de competencias conversacionales.

8. Considerar, en esas estrategias de cambio, las particularidades de cada universidad: su cultura, valores, experiencia, e historia singular de cada institución. Desde esta perspectiva ya no se plantearían visiones y misiones recursivas. Se estaría hablando, más bien, de la construcción de significados que serían modificados por las propias interrelaciones que ocurren en la organización, y entre la organización y su entorno, en una acción cooperativa y de apropiación social del conocimiento (1)

9. Reflexionar como acto investigativo esta aptitud unida a la criticidad y refutabilidad (Popper, 1967) en el dialogo con los otros que se produce al divulgar y difundir los resultados de investigaciones (10). Esto puede resultar un mecanismo propicio para fomentar el reciclaje de los métodos de investigación en los investigadores involucrados y el compartir esa experiencia con el cuerpo académico de la universidad de origen.

\section{Conclusiones}

Fortalecer el papel de transformación, de liderazgo del investigador universitario como actor social y agente de cambio implica redimensionar el carácter proactivo de la vinculación universidad-sector socio-productivo. No obstante los posibles obstáculos que se tengan en la ejecución de la actividad investigativa universitaria, es necesario, incluso en países como Venezuela, desarrollar, en medio de la crisis, un quehacer investigativo de compromiso ético con la propia realidad en respuesta al encargo o necesidades del entorno. Además, es preciso establecer una comunicación tal con ese entorno que permita el intercambio y la interacción proactiva de docencia- investigación-extensión en continua creación y movimiento, es decir, que la secuencia entre una actividad y otra, se encuentre hilada en representaciones dialécticas.

La innovación en la praxis investigativa universitaria requiere de la iniciativa e imaginación de sus colaboradores. En otros términos, precisa recrear permanentemente los métodos de investigación, adaptándolos al entorno, y no al revés, como se hace en la práctica ortodoxa de adaptar la realidad a la camisa de fuerza de métodos canónicos y prestablecidos. Entonces, se demanda una dinámica creativa y heterodoxa para así propiciar una praxis investigativa sustentable 
para explicar, comprender e interpretar una realidad que nos reclama un acto de observación que haría explicito lo implícito, para dar paso a una nueva Gestalt.

\section{REFERENCIAS}

AVERU (2016) Rectores universitarios exigen al Ejecutivo la asignación de un presupuesto justo, Recuperado de: http:// noticiaaldia.com/2016/05/rectores-universitarios-exigen-al-ejecutivo-asignacion-de-presupuesto-justo/ 30/07/2016

Banco Central de Venezuela (2014): Índice Nacional de Precios al Consumidor 2007-2014. Información Estadística BCV. Caracas: Banco Central de Venezuela e instituto Nacional de Estadística. http://www.bcv.org.ve/c2/indicadores.asp 10/02/2016

Ban-Ki-moon (2016) En Venezuela hay una crisis humanitaria. Recuperado de: http://www.el-nacional.com/GDA/ Ban-Ki-moon-Venezuela-crisis-humanitaria_0_901111941.html

Bertalanffy, L (1976). Teoría general de sistemas. Madrid: Fondo de Cultura Económica

Carvajal, B. (2001). Universidad y Prospectiva; Movilización de sus inteligencias. OMNIA. V. 7 (1 y 2) / 27-49/

Carvajal, B. (2005). “La universidad pública venezolana al inicio del siglo XXI ¿Una organización abierta al cambio?" Recuperado el 18 de marzo de 2016 de: http:// www.serbi.luz.edu.ve/tesis/index.php.

Carvajal, B. (2008). Universidad y Prospectiva. Aproximación Prospectiva a la Universidad Pública Venezolana al Inicio del siglo XXI. Revista Venezolana de Ciencias Sociales (RVCS). V. 12 (1) /11-40/
Carvajal, B. (2010a). La heterodoxia como alternativa metódica en la teoría y práctica investigativa universitaria. Reflexión acerca de los aportes que realizan en Venezuela Alejandro Moreno y Miguel Martínez. Espacio Abierto. Cuaderno Venezolano de Sociología. V.19, (1) /117-136/

Carvajal, B. (2010b). Inter y transdisciplinariedad en la Cultura Investigativa Universitaria. Cómo favorecer su práctica en la universidad venezolana. Revista Venezolana de Ciencias Sociales. UNERMB, V. 14 (2) / 452-467/

Carvajal, B. y Melgarejo. I. (2014) Métodos Creativos: uso de la teoría de las inteligencias múltiples en la praxis investigativa. Revista Latinoamericana de Multiciencias (RLM) V. 1 (1) /77-90/

Instituto Nacional de Estadística (INE) República Bolivariana de Venezuela. Índice Nacional de Precios al Consumidor. Variaciones Porcentuales 2008- Diciembre 2015. Recuperado de: http://www. ine.gov.ve/index.php?option $=$ com content\&view= category\&id=108\&Ite$\operatorname{mid}=62$ 20/08/2016

Informe 21 (2016) Francisco Faraco estima en $300 \%$ tasa real de inflación en el país. Recuperado de: https://informe21.com/ economia/francisco-faraco-estima-en300-tasa-real-de-inflacion-en-el-pais 30/08/2016

Kainnear T. \& Taylor J. (2003) Investigación de Mercado. Bogotá. Colombia: Mc Graw Hill

Mc Carthy, T. (2002) La Teoría Crítica de Jürgen Habermas. España, Madrid: Tecnos.

Martínez, M. (2009) Nuevos paradigmas en la investigación. Caracas: Alfa.

Martínez, M. (2004) Ciencia y Arte en Metodología Cualitativa D.F. México: Trillas 
Moreno, A. (1995) El aro y la trama. Episteme, modernidad y pueblo. Caracas: Centro de Investigaciones populares.

Moreno, A. (2006) La Investigación convivida. La experiencia vivida como horizonte epistemológico-práxico en la investigación en Ciencias Sociales. $\mathrm{He}$ terotopia. V. 11, (34) /9-26/

Moreau, A. (1999) Ejercicios y técnicas creativas de Gestalterapia. Málaga: Sirio.

Parra, M. (2008) Las intimidades de la academia. Un estudio cuanti-cualitativo sobre la dinámica de la profesión académica. Maracaibo: Universidad del Zulia

Perls, F. (1975) Yo, hambre y agresión. México: Fondo de cultura económica

Perls, F. (1977) Dentro y fuera del tarro de la basura. Santiago de Chile: Cuatro vientos

Popper, K. (1967) El desarrollo del conocimiento científico: conjeturas y refutaciones. Buenos Aires: Paidós

Ricoeur, P (1973) Ethics and Culture; Habermas and Gadamer in Dialogue, Philosophy Today.
Robine, JM (1998) Terapia Gestalt. Madrid. Gaia.

Rusque, A. (2003) De la diversidad a la unidad en la investigación cualitativa. Valencia, Venezuela: Vadel Hermanos.

Ritzer, G. (1993) Teoría Sociológica Contemporánea Madrid, España: Mc Graw-Hill.

Sautu, R. (2004) El método biográfico. La reconstrucción de la sociedad a partir del testimonio de los actores. Buenos Aires: Lumiere. Mead, George (1972) Espíritu, persona y sociedad. Argentina, Buenos Aires: Paidos

Savater F. y De Villena L. (1989) Heterodoxia y Contracultura, Barcelona, España: Montesinos.

UNESCO (1998) Declaración mundial sobre la educación superior en el siglo XXI: visión y acciónymarcodeacción prioritariaparaelcambio y el desarrollo de la educacion superior. Recuperado de: http://www.unesco.org/education/educprog/wche/declaration_spa. htm 03/03/2016

Vázquez, C. (2010), Borradores para la vida. Pensar y escribir sobre terapia Gestalt. México: La sociedad de Cultura Valle-Inclan. 